\title{
Comparative evaluation of copeptin and NT-proBNP in patients with severe acute decompensated heart failure, and prediction of adverse events in a 90-day follow-up period: A prospective clinical observation trial
}

\author{
JUN JIA $^{1 *}$, GUANG-LEI CHANG ${ }^{1 *}$, SHU QIN ${ }^{1}$, JIA CHEN ${ }^{1}$, WEN-YAN HE ${ }^{1}$, \\ KAI LU ${ }^{1}$, YONG LI ${ }^{2}$ and DONG-YING ZHANG ${ }^{1}$ \\ ${ }^{1}$ Department of Cardiology, The First Affiliated Hospital of Chongqing Medical University, Yuanjiagang, \\ Yuzhong, Chongqing 400016, P.R. China; ${ }^{2}$ Department of Basic Sciences, Center for Perinatal Biology, \\ Division of Pharmacology, Loma Linda University School of Medicine, Loma Linda, CA 92350, USA
}

Received August 16, 2015; Accepted October 21, 2016

DOI: $10.3892 /$ etm.2017.4111

\begin{abstract}
The present study compared the prognostic value of a marker, the C-terminal section of the arginine vasopressin prohormone (copeptin), with $\mathrm{N}$-terminal B-type natriuretic peptide (NT-proBNP) in patients with severe acute decompensated heart failure. A prospective, observational cohort study was conducted in a tertiary care hospital and enrolled 129 patients with severe acute decompensated heart failure. Clinicians were blinded to investigational markers except NT-proBNP, and the study participants were followed up for 90 days. The end-point was a composite of cardiovascular death or re-hospitalization due to decompensated heart failure. Of the 129 patients enrolled, 47 reached the end-point and 82 were in a stable condition during follow-up. Receiver operating characteristic curve analysis revealed that the areas under curve for the prediction of adverse events within 90 days were similar for copeptin $[0.602 \pm 0.052 ; 95 \%$ confidence interval (CI), 0.499-0.705], NT-proBNP $(0.659 \pm 0.048 ; 95 \%$ CI, 0.565-0.753) and their combination $(0.670 \pm 0.050 ; 95 \%$ CI, 0.573-0.767). Kaplan-Meier survival analysis showed that the predictive value of NT-proBNP regarding the probability of survival was superior compared with that of copeptin (log-rank test for trend, $\mathrm{P}=0.001$ vs. 0.040). Furthermore, multivariate Cox proportional-hazards regression analysis revealed that
\end{abstract}

Correspondence to: Dr Dong-Ying Zhang, Department of Cardiology, First Affiliated Hospital of Chongqing Medical University, 1 Friendship Road, Yuzhong, Chongqing 400016, P.R. China

E-mail: zdy.chris@gmail.com

*Contributed equally

Key words: heart failure, copeptin, N-terminal B-type natriuretic peptide, adverse events, mortality increased NT-proBNP and copeptin plasma concentrations were significant independent predictors of adverse events. The present study provided evidence that copeptin has similar predictive properties compared with NT-proBNP regarding adverse events within 90-days in patients with severe acute decompensated heart failure, but that copeptin may not provide superior 90-day prediction compared to NT-proBNP.

\section{Introduction}

Heart failure involves complex clinical symptoms and is a severe stage of various heart diseases, which has a high incidence and mortality. Incidence rate of adult is $1-2 \%$; mortality rates as high as $50 \%$ for five years. All diseases that cause chronic heart failure can lead to acute heart failure. As a result of the increasing number of patients with chronic heart failure, acute decompensated heart failure has become the predominant adverse event in patients with heart failure (1).

Acute decompensated heart failure is characterized by the first presentation or worsening of signs and symptoms of heart failure, leading to death or readmission. Biomarkers may support the diagnosis and prognosis of patients and may aid in their management. Previous studies have suggested that the N-terminal B-type natriuretic peptide (NT-proBNP) concentration increased in patients with heart failure, and that this may provide reliable diagnostic and prognostic information (2-5).

It is well established that neuroendocrine activation has a central role in the pathophysiology of heart failure, is extremely hazardous and results in myocardial injury in patients with heart failure if activation is sustained $(6,7)$. Other candidate peptides for the treatment of patients with heart failure should also be considered. One such peptide is arginine vasopressin (AVP), which is released from the hypovolaemia (8). AVP plasma values are elevated in patients with heart failure, and are associated with the severity of the disease (9). Copeptin, a peptide consisting of 39 amino acids, is a fragment of pre-pro-vasopressin that is synthesized and 
secreted in equimolar amounts to vasopressin (8). In contrast to AVP, copeptin is stable and can be easily measured $(10,11)$. A previous study revealed that copeptin is a strong prognostic biomarker for patients with chronic heart failure (12) and acute decompensated heart failure $(13,14)$.

The aim of the present study was to perform a head-to-head comparative evaluation of copeptin and NT-proBNP as predictors of 90-day mortality or recurrence in patients with severe acute decompensated heart failure.

\section{Materials and methods}

Study population. Between August 2011 and February 2012, the present study prospectively enrolled consecutive patients who had previously been referred to the Department of Cardiology of the First Affiliated Hospital of Chongqing Medical University (Chongqing, China) with acute heart failure. The Framingham Risk Score (15) for heart failure was used for the diagnosis of acute heart failure. The exclusion criteria were as follows: Patients below the age of 18 years or with a history of mental illness, coma, chronic renal insufficiency, acute renal insufficiency or currently receiving hemodialysis, previous tumors, the presence of acute myocardial infarction within the previous 3 months, significant pulmonary infectious diseases, liver disease or liver function abnormalities (increase of ammonia enzyme levels to twice the normal limit or above) or those who did not provide informed consent. The New York Heart Association (NYHA) functional classification was used to classify the level of cardiac function.

Only patients with severe acute decompensated heart failure, who were confirmed to have NYHA functional class III or IV by two senior cardiologists simultaneously, and those who had completed at least a 90-day period of follow-up were enrolled. A total of 129 patients with severe acute decompensated heart failure were analyzed in the present study.

The study was conducted according to the Declaration of Helsinki and was approved by the Chongqing Medical University Review Board (Chongqing, China). All participants provided written informed consent.

Follow-up and study end-points. Clinical follow-up was usually performed either during outpatient clinic visits each month or by telephone interviews. The pre-defined end-point of the follow-up investigation was defined as cardiovascular death or re-hospitalization due to decompensated heart failure. Mortality and re-hospitalization data were obtained from hospital medical records and/or by telephone contact with the patients' relatives. All study participants were followed up for a minimum of 90 days from the time-point they were diagnosed at our department.

Clinical and laboratory evaluation. Demographic and clinical data, including the NYHA functional class, were obtained. During the initial patient examination, blood samples were taken from an antecubital vein for the measurement of NT-proBNP concentrations and were analyzed within $4 \mathrm{~h}$ using a commercially available assay (Roche Elecsys proBNP Immunoassay; Roche Diagnostics, Basel, Switzerland). Aliquots of the collected blood samples $(0.6 \mathrm{TIU} / \mathrm{ml})$ were loaded into plastic tubes containing EDTA and aprotinin. The tubes were placed on ice prior to centrifugation at $1,600 \times \mathrm{x}$ for $15 \mathrm{~min}$ at $4^{\circ} \mathrm{C}$ to collect the plasma, which was stored at $-70^{\circ} \mathrm{C}$ until it was used in assays in 1 batch at $\sim 9$ months after collection. Researchers were blinded to the clinical features and biochemical data during assays. No sample was thawed $>2$ times. Copeptin was detected with a novel commercial chemiluminescence assay [EK-065-32 Copeptin (Human); Phoenix Pharmaceuticals Inc., Burlingame, CA, USA]. After a fasting period of at least $12 \mathrm{~h}$, blood samples were drawn to examine blood counts, lipid parameters, high sensitive $\mathrm{C}$-reactive protein (hs-CRP), renal function parameters and hepatic function.

Statistical analysis. The normal distribution of continuous variables was expressed as the mean \pm standard deviation. Skewed distribution variables were expressed as medians and interquartile ranges, or sums and percentages. Differences between groups for normally distributed continuous variables were analyzed using an independent-samples t-test. The Mann-Whitney U test was employed when continuous variables were not normally distributed. Categorical variables were tested using Pearson's Chi-square test. Spearman's rank correlation was used to assess the association between variables. To evaluate the prediction accuracy of adverse events, receiver operating characteristic (ROC) plots were constructed and areas under the curves (AUCs) were calculated for plasma NT-proBNP, copeptin and their combination. AUCs were compared according to the method by Hanley and McNeil (16). The entire study population $(n=129)$ was then stratified on the basis of cut-off concentrations for the two analytes. Cut-off concentrations were determined according to the 'Youden index' derived directly from the ROC curves. Kaplan-Meier estimates of the distribution of times from baseline to the end-point were computed and log-rank tests to determine trends were performed to compare the survival curves between the groups. Furthermore, Cox proportional hazards regression was used to analyze the effect of several confounding risk factors on survival. Initially, univariate Cox-regression analysis was performed to estimate the impact of risk factors on the survival of patients. Subsequently a multivariate Cox regression analysis was performed using the forwards conditional method, which was adjusted to the patients' confounding risk factors. All data were analyzed using SPSS statistical software (version 17.0; SPSS, Inc., Chicago, IL, USA), and the MedCalc package (version 11.5.0.0; MedCalc Software bvba, Ostend, Belgium). All probabilities were two-tailed and $\mathrm{P}<0.05$ was considered to indicate a significant difference.

\section{Results}

A total of 129 patients were included in the present study, and etiological classifications of the patients are presented in Table I. The median age was 73 years (range, 64.5-79.5 years), the number of males was $54(41.9 \%), 44$ patients $(34.1 \%)$ had a history of smoking, $76(58.9 \%)$ had hypertension, 37 (28.7\%) were diabetics, 19 (14.7\%) had a history of chronic obstructive pulmonary disease and 59 (45.7\%) had NYHA functional class IV status. Of the 129 patients who completed the 90-day follow-up, $82(63.6 \%)$ were in a stable condition 
Table I. Etiological classification of patients.

\begin{tabular}{lccc}
\hline Etiological classification & $\begin{array}{c}\text { Total patients, } \\
\mathrm{n}(\%)\end{array}$ & $\begin{array}{c}\text { Patients with } \\
\text { Stable condition, } \mathrm{n}(\%)\end{array}$ & $\begin{array}{c}\text { Patients reaching } \\
\text { adverse end-point, } \mathrm{n}(\%)\end{array}$ \\
\hline Coronary arteriosclerosis & $41(31.8)$ & $26(20.2)$ & $15(11.6)$ \\
Hypertensive heart disease & $40(31.0)$ & $30(23.2)$ & $10(7.8)$ \\
Dilated cardiomyopathy & $25(19.4)$ & $14(10.9)$ & $11(8.5)$ \\
Rheumatic heart disease & $12(9.3)$ & $7(5.4)$ & $5(3.9)$ \\
Senile valvular disease & $4(3.1)$ & $3(2.3)$ & $1(0.7)$ \\
Hyperthyroid cardiomyopathy & $3(2.3)$ & $2(1.6)$ & $1(0.7)$ \\
Pulmonary heart disease & $2(1.6)$ & $0(0)$ & $2(2.6)$ \\
Unknown causes & $2(1.6)$ & $0(0)$ & $2(1.6)$ \\
\hline
\end{tabular}

and $47(36.4 \%)$ reached an adverse end-point within 90 days from the time they were enrolled.

Table II shows the baseline demographics and clinical characteristics according to the 90-day follow-up result categories. Patients who reached an adverse end-point showed a higher NYHA III/IV status, NT-proBNP and copeptin levels compared with patients in a stable condition, while there were no significant differences between the two groups in terms of length of hospital stay, left ventricular ejection fraction and general demographic characteristics. The results of correlation analyses for the plasma copeptin levels and other factors are displayed in Table III. It was shown that copeptin concentration was positively correlated with the age of the patients and the grading of NYHA classification.

Copeptin and NT-proBNP levels, stratified by NYHA classification, are shown in Fig. 1. In patients with NYHA class III, the two markers were significantly lower compared with those in patients with NYHA class IV $(\mathrm{P}<0.01)$.

The ROC curves are shown in Fig. 2. With regard to patients who reached the end-point and those who were in a stable condition, the AUC for copeptin was $0.602 \pm 0.052$ (95\% CI, 0.499-0.705), that for NT-proBNP was $0.659 \pm 0.048$ (95\% CI, 0.565-0.753) and that of their combination was $0.670 \pm 0.050$ (95\% CI, 0.573-0.767). Comparison of the ROC curves revealed no significant differences between the AUCs for NT-proBNP and copeptin $(\mathrm{P}=0.414)$. Therefore, with regard to the prediction of 90-day survival, the combination of copeptin and NT-proBNP was not superior to each single marker alone $(\mathrm{P}>0.05)$.

Table IV depicts the specificity, sensitivity and prognostic value of NT-proBNP and copeptin regarding 90-day adverse end-points above their cutoff concentrations for the study's sample $(n=129)$. Figs. 3 and 4 show the Kaplan-Meier curves for the 129 patients with severe acute decompensated heart failure who were stratified into two groups according to the cutoff concentrations of NT-proBNP and copeptin. The survival rate was significantly lower in patients with increased baseline plasma NT-proBNP and copeptin concentrations.

The results of the Cox proportional-hazards regression analysis are shown in Table V. According to univariate analyses, NT-proBNP and copeptin were associated with a significant risk with the presence of NYHA class III/IV at baseline being a possible confounding factor. Significant predictors for the adverse end-point of severe acute decompensated heart failure are summarized in Table V. Multivariate Cox proportional hazards regression analysis indicated that the copeptin concentration [risk ratio (RR), 1.956; 95\% CI, 1.048-3.648; $\mathrm{P}=0.035]$ and the NT-proBNP concentration (RR, 4.415; 95\% $\mathrm{CI}, 1.357-14.358 ; \mathrm{P}=0.014)$ were independently associated with adverse end-points.

The survival probability of patients with different serum concentration level of NT-proBNP and copeptin is shown in Fig. 5. As shown in Fig. 5, patients with the lower level of NT-proBNP $(<1,471.50 \mathrm{pg} / \mathrm{ml})$ and copeptin $(<0.89 \mathrm{ng} / \mathrm{ml})$ had the highest survival probability. Neither a higher level of NT-proBNP or copeptin could lead to the increase of mortality. Furthermore, patients with the higher level of NT-proBNP $(>1,471.50 \mathrm{pg} / \mathrm{ml})$ and copeptin $(>0.89 \mathrm{ng} / \mathrm{ml})$ reach the highest mortality in the four groups at the end of follow-up time (log rank test, $\mathrm{P}<0.05)$.

\section{Discussion}

The the major conclusion of the present study is that increased concentrations of copeptin and NT-proBNP determined in plasma samples drawn from patients with severe acute decompensated heart failure at the time of initial presentation indicate an increased risk of mortality and re-hospitalization. Patients with high plasma concentrations of copeptin and NT-proBNP exhibited higher rates of poor outcome independent of other confounders, including advanced age, systolic dysfunction and NYHA class III/IV at baseline in the sample investigated. For patients in the severe stage of heart failure, copeptin was an independent predictive factor; however, it was not superior to NT-proBNP.

The majority of single-variable markers are characterized by the unsatisfactory discrimination of patients with and without increased mortality due to heart failure. By contrast, NT-proBNP has been shown to be a useful marker for risk stratification and prognosis in the setting of heart failure, and the present study showed similar results $(17,18)$.

In the present study, copeptin levels were significantly higher in patients with higher NYHA classes, as well as in patients who reached the adverse end-point. However, the stimuli resulting in increased secretion of vasopressin in patients with heart failure have yet to be elucidated. Several previous studies have assessed the utility of copeptin as 
Table II. Demographics and clinical characteristics of patients at follow-up.

\begin{tabular}{|c|c|c|c|}
\hline Variable & $\begin{array}{l}\text { Patients in a stable } \\
\text { condition }(n=82)\end{array}$ & $\begin{array}{l}\text { Patients reaching the } \\
\text { adverse end-point }(n=47)\end{array}$ & P-value \\
\hline \multicolumn{4}{|l|}{ Demographics } \\
\hline Age (years) & $73(66-79)$ & $74(58-82)$ & 0.743 \\
\hline Male gender & $33(40.2)$ & $21(44.7)$ & 0.624 \\
\hline Smoking & $30(36.6)$ & $14(29.8)$ & 0.435 \\
\hline BMI $\left(\mathrm{kg} / \mathrm{m}^{2}\right)$ & $22.7 \pm 3.0$ & $21.9 \pm 2.9$ & 0.129 \\
\hline \multicolumn{4}{|l|}{ Co-morbidities } \\
\hline Hypertension & $52(63.4)$ & $24(51.1)$ & 0.172 \\
\hline Diabetes & $27(32.9)$ & $10(21.3)$ & 0.161 \\
\hline COPD & $14(17.1)$ & $5(10.6)$ & 0.323 \\
\hline \multicolumn{4}{|l|}{ NYHA class } \\
\hline NYHA III & $51(62.2)$ & $19(40.4)$ & 0.017 \\
\hline NYHA IV & $31(37.8)$ & $28(59.6)$ & \\
\hline LVEF & $52.0(39.8-62.0)$ & $49.5(39.8-57.8)$ & 0.354 \\
\hline \multicolumn{4}{|c|}{ Length of hospital stay (days) } \\
\hline Total & $8(6-13)$ & $9(4-14)$ & 0.763 \\
\hline In ICU & $0(0-2)$ & $1(0-3)$ & 0.103 \\
\hline \multicolumn{4}{|l|}{ Biochemical parameters } \\
\hline WBC $\left(\times 10^{9}\right)$ & $6.8(5.4-8.2)$ & $6.6(4.7-8.5)$ & 0.676 \\
\hline Hemoglobin (g/l) & $126.5 \pm 21.7$ & $119.2 \pm 19.8$ & 0.067 \\
\hline LDL-C (mmol/l) & $2.1(1.6-2.8)$ & $1.9(1.5-2.2)$ & 0.063 \\
\hline TG (mmol/l) & $1.0(0.8-1.6)$ & $1.1(0.8-1.3)$ & 0.292 \\
\hline hs-CRP (mg/l) & $6.4(1.6-14.5)$ & $8.6(2.6-19.6)$ & 0.209 \\
\hline Serum sodium $(\mathrm{mmol} / \mathrm{l})$ & $139.0(136.0-141.0)$ & $139.0(136.0-141.0)$ & 0.994 \\
\hline hs-cTNT (pg/ml) & $24.0(10.0-41.0)$ & $29.0(16.0-59.5)$ & 0.118 \\
\hline MYO (ng/ml) & $53.0(34.8-82.3)$ & $46.6(29.0-66.7)$ & 0.631 \\
\hline CK-MB (ng/ml) & $2.7(1.9-3.6)$ & $2.8(2.2-3.5)$ & 0.470 \\
\hline \multicolumn{4}{|l|}{ Biomarkers values } \\
\hline NT-proBNP (pg/ml) & 3275 (1008.8-8827.0) & 6656 (2919.0-15701.0) & 0.003 \\
\hline Copeptin (ng/ml) & $0.79 \pm 0.30$ & $0.92 \pm 0.31$ & 0.022 \\
\hline
\end{tabular}

Table III. Correlation between plasma copeptin levels and other factors in the entire study sample $(n=129)$.

\begin{tabular}{|c|c|c|c|c|}
\hline \multirow[b]{2}{*}{ Parameter } & \multicolumn{2}{|c|}{ Copeptin } & \multicolumn{2}{|c|}{ NT-proBNP } \\
\hline & $\mathrm{r}$ & P-value & $\mathrm{r}$ & P-value \\
\hline Age & 0.183 & 0.038 & -0.121 & 0.172 \\
\hline NYHA classification & 0.283 & 0.001 & 0.497 & $<0.001$ \\
\hline LVEF & 0.024 & 0.803 & -0.572 & $<0.001$ \\
\hline
\end{tabular}

Spearman's correlation coefficients for the study parameters. NYHA, New York Heart Association; LVEF, left ventricular ejection fraction. a prognostic biomarker for a variety of other indications, including hemorrhagic and septic shock (10), exacerbations of chronic obstructive pulmonary disease (19), certain respiratory tract infections $(16)$, stroke $(20,21)$ and traumatic brain injury (22). It may be hypothesized that the body responds to emergency and severe diseases by the instantaneous release of immediate AVP and copeptin. The use of copeptin as a biomarker appears to be indicative of the patients' stress levels (23). For patients with severe-stage heart failure (NYHA functional classes III and IV), copeptin provided independent information; however, it was not the marker with the highest predictive value and was inferior to NT-proBNP. An explanation for this finding may be that the ability of biomarkers to predict outcomes is not static, but rather varies over time (24). 
Table IV. Diagnostic information for the prediction of adverse events within 90 days by NT-proBNP and copeptin in the entire study sample $(\mathrm{n}=129)$.

\begin{tabular}{lcccc}
\hline Cutoff concentration $(\mathrm{pg} / \mathrm{ml})$ & Sensitivity, \% (95\% CI) & Specificity, \% (95\% CI) & PPV, \% (95\% CI) & NPV, \% (95\% CI) \\
\hline NT-proBNP: 1471.5 & $93.6(82.5-98.7)$ & $32.9(22.9-44.2)$ & $44.4(34.5-54.8)$ & $90.0(73.1-98.0)$ \\
Copeptin: 890.0 & $46.8(32.1-61.9)$ & $69.5(58.4-79.2)$ & $46.8(32.1-61.9)$ & $69.5(58.3-79.3)$ \\
\hline
\end{tabular}

CI, confidence interval; NPV, negative predictive value; PPV, positive predictive value; NT-proBNP, N-terminal pro-B-type natriuretic peptide.
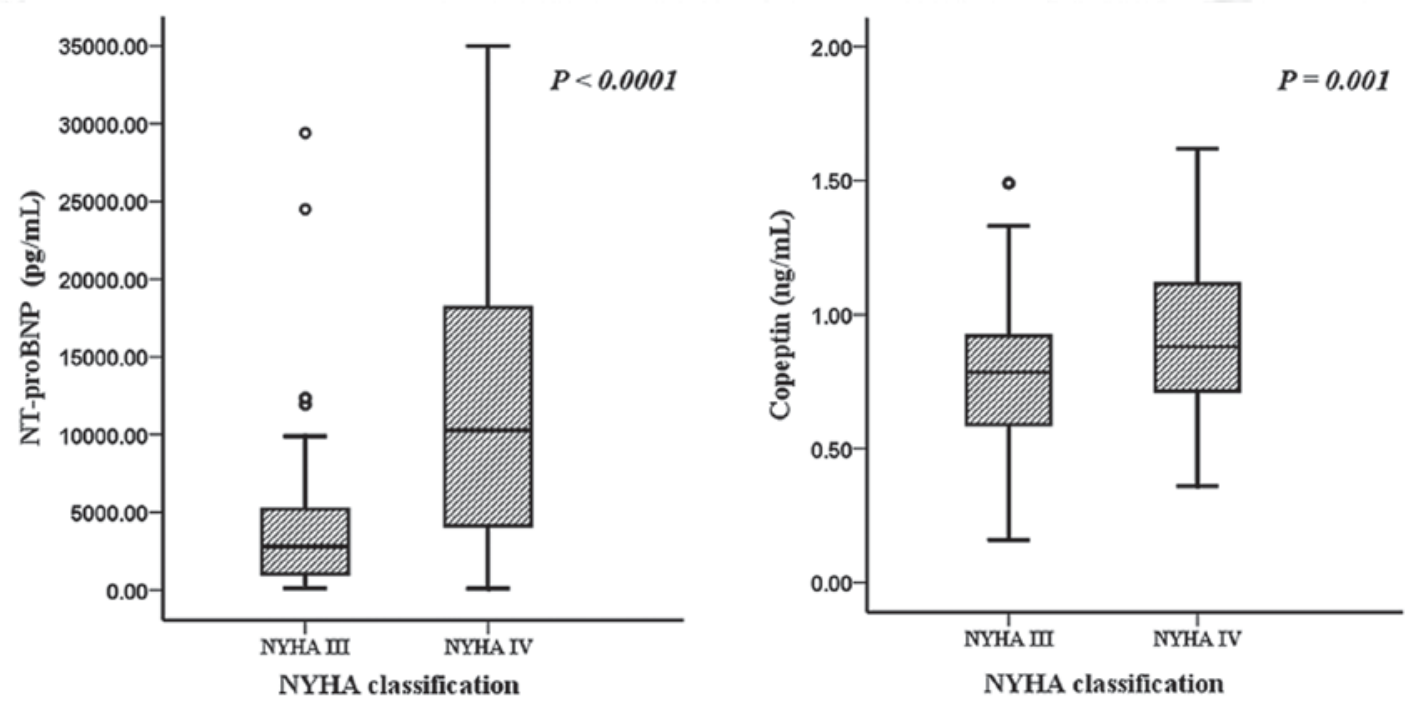

Figure 1. Box plots for levels of NT-proBNP in patients classified as NYHA III/IV at the time of presentation. Solid horizontal lines denote median values, boxes represent $25-75$ th percentiles and bars indicate the range, while circles denote outliers. Statistically significant differences: Copeptin (P=0.001 for NYHA class III vs IV) and NT-proBNP (P<0.0001 for NYHA class III vs IV). NT-proBNP, N-terminal pro-B-type natriuretic peptide; NYHA, New York Heart Association.

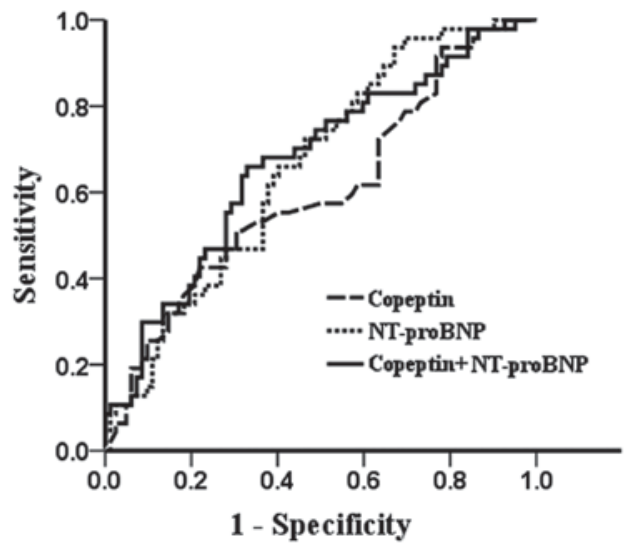

Figure 2. ROC plot indicating the ability of copeptin, NT-proBNP and their combination to predict the 90 -day adverse events in 129 patients with acute destabilized heart failure ( 82 without vs. 47 with end-point); the area under the curve was 0.602 for copeptin, 0.659 for NT-proBNP and 0.670 for their combination. ROC, receiver operating characteristic; NT-proBNP, $\mathrm{N}$-terminal pro-B-type natriuretic peptide.

Previous studies have shown that copeptin had initially strong prognostic abilities, but became slightly less accurate (24). By contrast, natriuretic peptides, which were initially poor acute prognostic markers, showed improved performance during the follow-up period, suggesting that markers reflecting the hemodynamic status or myocardial necrosis have superior short-term prognostic value, which however deteriorates following the resolution of the acute insult.

While the present study was not an interventional trial, the results demonstrated that the patients who reached the adverse end-point did not have an increased length of hospital stay. The period spent in hospital (in days) showed no significant difference with regard to stays in a regular ward or intensive care unit, suggesting that in future clinical practice, high-risk patients should receive additional care. It is important to note that, while elevated copeptin or NT-proBNP levels are associated with the risk of adverse end-points, severe acute decompensated heart failure treatment should not solely depend on biomarkers, as these are hemodynamic markers that are not specific to heart failure (25). However, a high concentration of either of these markers appears to be an indication of an increased risk of adverse events and poor outcome.

The present study had several potential limitations. One major limitation was that the study only assessed a relatively small number of patients and was single-centered. Thus, the results may not accurately represent the general demographics of patients with severe acute decompensated heart failure in other institutions. Therefore, the reproduction of the results 
Table V. Results of univariate Cox proportional-hazards regression analyzing the effect of baseline variables on adverse end-points.

\begin{tabular}{llllll}
\hline & \multicolumn{2}{c}{ Univariate analysis } & & \multicolumn{2}{c}{ Mutivariate analysis } \\
\cline { 2 - 3 } Baseline variable & Risk ratio (95\% CI) & P-value & & Risk ratio (95\% CI) & P-value \\
\hline Copeptin $(\geq 0.89 \mathrm{ng} / \mathrm{ml})$ & $1.800(1.015-3.190)$ & 0.044 & & $1.956(1.048-3.648)$ & 0.035 \\
NT-proBNP $(\geq 1471.50 \mathrm{pg} / \mathrm{ml})$ & $5.611(1.741-18.080)$ & 0.004 & & $4.415(1.357-14.358)$ & 0.014 \\
NYHA classes III/IV & $2.042(1.140-3.658)$ & 0.016 & & NS \\
Advanced age & $1.496(0.810-2.762)$ & 0.198 & & \\
Systolic dysfunction & $1.027(0.502-2.102)$ & 0.941 & & \\
\hline
\end{tabular}

Advanced age was defined as age $\geq 80$ years; systolic dysfunction was defined as left ventricular ejection fraction $\leq 40 \%$. NT-proBNP, N-terminal pro-B-type natriuretic peptide; NYHA, New York Heart Association; CI, confidence interval; NS, not significant.

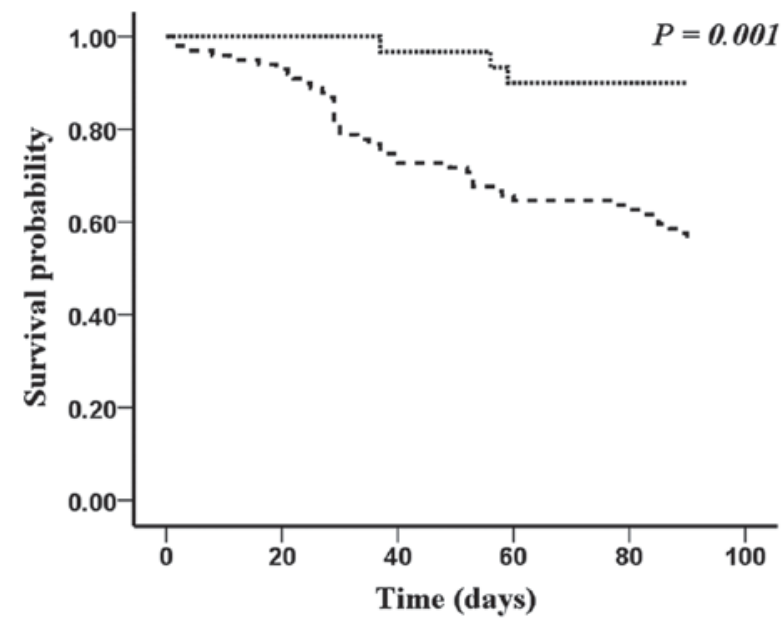

Figure 3. Kaplan-Meier plots showing the proportion of patients in a stable condition out of the 129 patients with severe acute destabilized heart failure who were stratified into two groups according to plasma NT-proBNP cutoff point at baseline (dotted line, first tercile $<1471.50 \mathrm{pg} / \mathrm{ml}, \mathrm{n}=30$; dashed line, 2nd tercile $\geq 1471.50 \mathrm{pg} / \mathrm{ml}, \mathrm{n}=99$; log-rank test for trend, $\mathrm{P}=0.001$ ). $\mathrm{N}$-terminal pro-B-type natriuretic peptide. NT-proBNP, N-terminal pro-B-type natriuretic peptide.

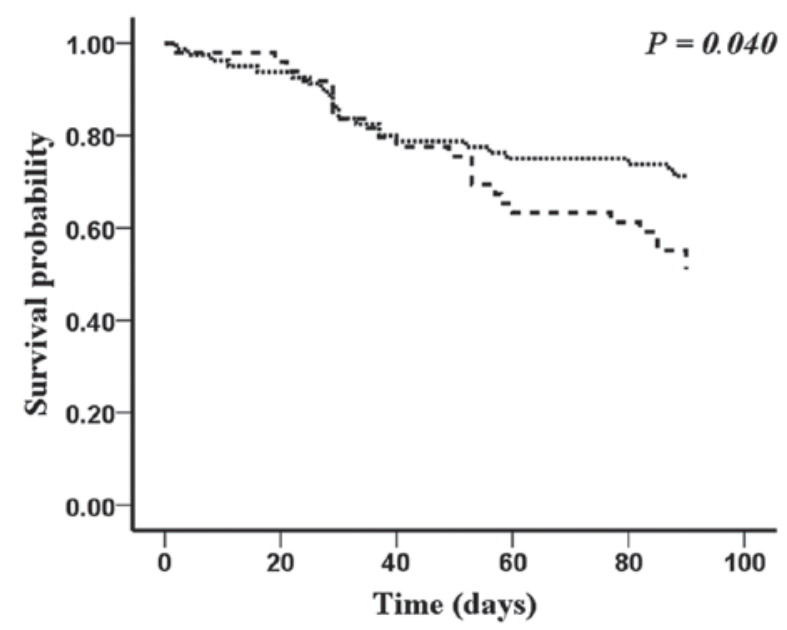

Figure 4. Kaplan-Meier plots showing the proportion of patients in a stable condition of the 129 patients with severe acute destabilized heart failure who were stratified into two groups according to plasma copeptin cutoff point at baseline (dotted line, first tercile $<0.89 \mathrm{ng} / \mathrm{ml}, \mathrm{n}=80$; dashed line, 2nd tercile $\geq 0.89 \mathrm{ng} / \mathrm{ml}, \mathrm{n}=49$; $\log$-rank test for trend, $\mathrm{P}=0.040$ ).

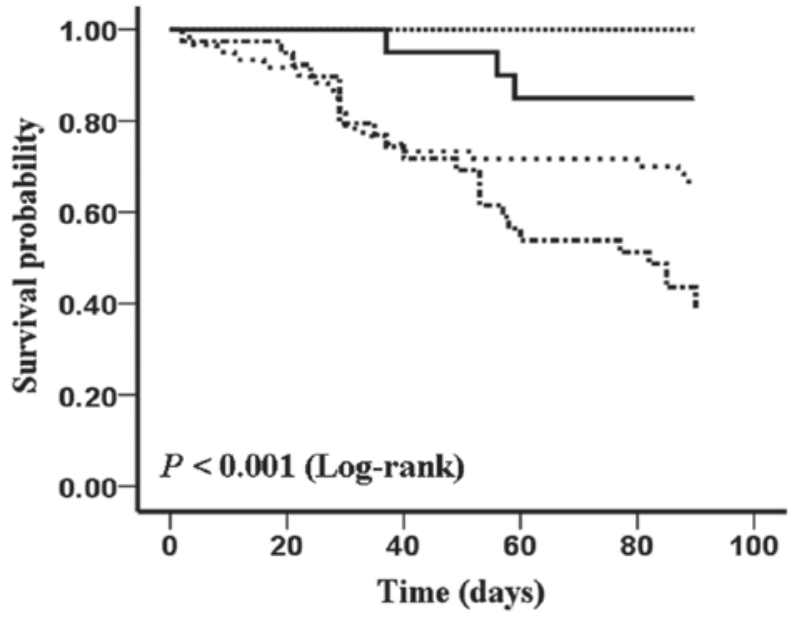

Figure 5. Kaplan-Meier plots showing the proportion of patients in a stable condition out of the 129 patients with severe acute destabilized heart failure who were stratified into two groups according to plasma NT-proBNP and copeptin cutoff point at baseline (straight line, first tercile $<1471.50 \mathrm{pg} / \mathrm{ml}$ for NT-proBNP and first tercile $<0.89 \mathrm{ng} / \mathrm{ml}$ for copeptin; narrow dotted line, first tercile $<1471.50 \mathrm{pg} / \mathrm{ml}$ for NT-proBNP and 2 nd tercile $\geq 0.89 \mathrm{ng} / \mathrm{ml}$ for copeptin; wide dotted line, 2nd tercile $<1471.50 \mathrm{pg} / \mathrm{ml}$ for NT-proBNP and first tercile $\geq 0.89 \mathrm{ng} / \mathrm{ml}$ for copeptin; dot-dashed line, second tercile $\geq 1471.50 \mathrm{pg} / \mathrm{ml}$ for NT-proBNP and 2 nd tercile $\geq 0.89 \mathrm{ng} / \mathrm{ml}$ for copeptin; $\mathrm{P}<0.001)$. NT-proBNP, N-terminal pro-B-type natriuretic peptide.

of the present study in other centers or by multicenter studies may provide a stronger argument for their validity. A further limitation is that during follow-up, due to personal economic problems, the treatment of certain patients was not in accordance with best clinical practice or in strict accordance with guidelines. Thus, the treatment modalities during follow-up may have affected the results. Furthermore, a number of parameters [blood pressure and renal function, which have consistently demonstrated prognostic value (26)] have not been included in the analysis of the present study. Finally, copeptin was measured separately, using different standardized evaluations. Although reflective of real-world clinical practice, comparison to other studies may indicate a limitation in the copeptin portion of this analysis.

In conclusion, the results of the present study suggested that NT-proBNP and copeptin measurements may have similar predictive value regarding adverse events occurring within 90 
days of severe acute decompensated heart failure. However, copeptin may provide inferior 90-day prediction compared with NT-proBNP. Future studies evaluating the role of these biomarkers are warranted and may further improve the prediction of clinical outcome.

\section{Acknowledgements}

The content of the present study was presented at the 24th Great Wall International Congress of Cardiology/Asia Pacific Heart Congress/International Congress of Cardiovascular Prevention and Rehabilitation in Beijing, P.R. China, 10-13.10.2013, pp E218-E219. The present study was supported by National Natural Science Fund (grant no. 81570212) and the Natural Science Foundation Project of CQ CSTC (grant no. CSTC, 2011jjA10008). Chongqing Municipal Health Bureau fund (grant nos. 010-1-07, 2012-2-125 and ZY20132124) and the National key Clinical Specialties Construction Program of China (grant no. 2011-170).

\section{References}

1. Cowie MR, Mosterd A, Wood DA, Deckers JW, Poole-Wilson PA, Sutton GC and Grobbee DE: The epidemiology of heart failure. Eur Heart J 18: 208-225, 1997.

2. Tsutamoto T, Wada A, Maeda K, Hisanaga T, Maeda Y, Fukai D, Ohnishi M, Sugimoto Y and Kinoshita M: Attenuation of compensation of endogenous cardiac natriuretic peptide system in chronic heart failure: Prognostic role of plasma brain natriuretic peptide concentration in patients with chronic symptomatic left ventricular dysfunction. Circulation 96: 509-516, 1997.

3. Januzzi JL, van Kimmenade R, Lainchbury J, Bayes-Genis A, Ordonez-Llanos J, Santalo-Bel M, Pinto YM and Richards M: NT-proBNP testing for diagnosis and short-term prognosis in acute destabilized heart failure: An international pooled analysis of 1256 patients: The international collaborative of NT-proBNP study. Eur Heart J 27: 330-337, 2006.

4. Moe GW, Howlett J, Januzzi JL and Zowall H; Canadian Multicenter Improved Management of Patients With Congestive Heart Failure (IMPROVE-CHF) Study Investigators: N-terminal pro-B-type natriuretic peptide testing improves the management of patients with suspected acute heart failure: Primary results of the Canadian prospective randomized multicenter IMPROVE-CHF study. Circulation 115: 3103-3110, 2007.

5. Maisel A, Mueller C, Adams K Jr, Anker SD, Aspromonte N, Cleland JG, Cohen-Solal A, Dahlstrom U, DeMaria A, Di Somma S, et al: State of the art: Using natriuretic peptide levels in clinical practice. Eur J Heart Fail 10: 824-839, 2008.

6. Braunwald E: Biomarkers in heart failure. N Engl J Med 358: 2148-2159, 2008.

7. Fiore G, Suppress P, Triggiani V, Resta F and Sabba C: Neuroimmune activation in chronic heart failure. Endocr Metab Immune Disord Drug Targets 13: 68-75, 2013.

8. Land H, Schütz G, Schmale H and Richter D: Nucleotide sequence of cloned cDNA encoding bovine arginine vasopressin-neurophysin II precursor. Nature 295: 299-303, 1982.

9. Friedmann AS, Malott KA, Memoli VA, Pai SI, Yu XM and North WG: Products of vasopressin gene expression in small-cell carcinoma of the lung. Br J Cancer 69: 260-263, 1994.

10. Morgenthaler NG, Müller B, Struck J, Bergmann A, Redl H and Christ-Crain M: Copeptin, a stable peptide of the arginine vasopressin precursor, is elevated in hemorrhagic and septic shock. Shock 28: 219-226, 2007.

11. Morgenthaler NG, Struck J, Alonso C and Bergmann A: Assay for the measurement of copeptin, a stable peptide derived from the precursor of vasopressin. Clin Chem 52: 112-119, 2006.
12. Stoiser B, Mörtl D, Hülsmann M, Berger R, Struck J, Morgenthaler NG, Bergmann A and Pacher R: Copeptin, a fragment of the vasopressin precursor, as a novel predictor of outcome in heart failure. Eur J Clin Invest 36: 771-778, 2006.

13. Gegenhuber A, Struck J, Dieplinger B, Poelz W, Pacher R, Morgenthaler NG, Bergmann A, Haltmayer M and Mueller T: Comparative evaluation of $\mathrm{B}$-type natriuretic peptide, mid-regional pro-A-type natriuretic peptide, mid-regional pro-adrenomedullin, and Copeptin to predict 1-year mortality in patients with acute destabilized heart failure. J Card Fail 13: 42-49, 2007.

14. Maisel A, Xue Y, Shah K, Mueller C, Nowak R, Peacock WF, Ponikowski P, Mockel M, Hogan C, Wu AH, et al: Increased 90-day mortality in patients with acute heart failure with elevated copeptin: Secondary results from the Biomarkers in Acute Heart Failure (BACH) study. Circ Heart Fail 4: 613-620, 2011.

15. Dickstein K, Cohen-Solal A, Filippatos G, McMurray JJ, Ponikowski P, Poole-Wilson PA, Strömberg A, van Veldhuisen DJ, Atar D, Hoes AW, et al: ESC Guidelines for the diagnosis and treatment of acute and chronic heart failure 2008: Rhe Task Force for the Diagnosis and Treatment of Acute and Chronic Heart Failure 2008 of the European Society of Cardiology. Developed in collaboration with the Heart Failure Association of the ESC (HFA) and endorsed by the European Society of Intensive Care Medicine (ESICM). Eur Heart J 29: 2388-2442, 2008.

16. Müller B, Morgenthaler N, Stolz D, Schuetz P, Müller C, Bingisser R, Bergmann A, Tamm M and Christ-Crain M: Circulating levels of copeptin, a novel biomarker, in lower respiratory tract infections. Eur J Clin Invest 37: 145-152, 2007.

17. Noveanu M, Breidthardt T, Potocki M, Reichlin T, Twerenbold R, Uthoff H, Socrates T, Arenja N, Reiter M, Meissner J, et al: Direct comparison of serial B-type natriuretic peptide and NT-proBNP levels for prediction of short- and long-term outcome in acute decompensated heart failure. Critical Care 15: R1, 2011.

18. Metra M, Nodari S, Parrinello G, Specchia C, Brentana L, Rocca P, Fracassi F, Bordonali T, Milani P, Danesi R, et al: The role of plasma biomarkers in acute heart failure. Serial changes and independent prognostic value of NT-proBNP and cardiac troponin-T. Eur J Heart Fail 9: 776-786, 2007.

19. Hanley JA and McNeil BJ: A method of comparing the areas under receiver operating characteristic curves derived from the same cases. Radiology 148: 839-843, 1983.

20. Katan M, Nigro N, Fluri F, Schuetz P, Morgenthaler NG, Jax F, Meckel S, Gass A, Bingisser R, Steck A, et al: Stress hormones predict cerebrovascular re-events after transient ischemic attacks. Neurology 76: 563-566, 2011.

21. Urwyler SA, Schuetz P, Fluri F, Morgenthaler NG, Zweifel C, Bergmann A, Bingisser R, Kappos L, Steck A, Engelter S, et al: Prognostic value of copeptin: One-year outcome in patients with acute stroke. Stroke 41: 1564-1567, 2010.

22. Dong XQ, Huang M, Yang SB, Yu WH and Zhang ZY: Copeptin is associated with mortality in patients with traumatic brain injury. J Trauma 71: 1194-1198, 2011.

23. Katan M, Morgenthaler N, Widmer I, Puder JJ, König C, Müller B and Christ-Crain M: Copeptin, a stable peptide derived from the vasopressin precursor, correlates with the individual stress level. Neuro Endocrinol Lett 29: 341-346, 2008.

24. Peacock WF, Nowak R, Christenson R, DiSomma S, Neath SX, Hartmann O, Mueller C, Ponikowski P, Möckel M, Hogan C, et al: Short-term mortality risk in emergency department acute heart failure. Acad Emerg Med 18: 947-958, 2011.

25. Nickel CH, Bingisser R and Morgenthaler NG: The role of copeptin as a diagnostic and prognostic biomarker for risk stratification in the emergency department. BMC Medicine 10: 7, 2012.

26. Fonarow GC, Adams KF Jr, Abraham WT, Yancy CW and Boscardin WJ; ADHERE Scientific Advisory Committee, Study Group, and Investigators: Risk stratification for in-hospital mortality in acutely decompensated heart failure: Classification and regression tree analysis. JAMA 293: 572-580, 2005. 